\title{
Concomitant Anomalous Branching of Facial Nerve and Double Parotid Duct: A Case Report
}

\author{
Amro Mohamed Soliman, Elvy Suhana Ramli, Srijit Das, and Norzana Abd Ghafar \\ Department of Anatomy, Universiti Kebangsaan Malaysia Medical Centre, 56000 Kuala Lumpur, Malaysia.
}

\begin{abstract}
The facial nerve divides within the parotid gland into upper temporozygomatic and lower cervicofacial branches. The two branches further subdivide and emerge from the parotid gland as five main branches. We observed a rare anomalous branching pattern of the facial nerve along with double parotid duct on the right side of a 50-year-old male cadaver. The two parotid ducts emerged at the level of the anterior border of parotid gland then united to form one single duct thereby opened into the oral cavity. The first duct (D1) emerged from the upper one third of the anterior border of the parotid gland and traversed horizontally for $9 \mathrm{~mm}$ to join the second duct. Knowledge of anomalous branching pattern of facial nerve and double parotid ducts may be beneficial for maxillofacial surgeons.
\end{abstract}

Keywords: Anomaly, facial nerve, branches, parotid duct, face.

\section{INTRODUCTION}

Facial nerve exits the cranium through the stylomastoid foramen which is located between the mastoid and styloid processes. ${ }^{1}$ The facial nerve is liable to be injured in its extratemporal course as a result of facial trauma and laceration, or during any surgery involving the parotid, submandibular glands or temporomandibular joint. ${ }^{2}$ Knowledge of the topographical anatomy and surgical landmarks that aid in locating its branches is essential for the nerve is to be preserved during any surgery. The facial nerve and its branches are known to exhibit many variations.

Standard textbook of anatomy describes the main trunk of facial nerve to traverse anteriorly and enter the parotid gland (PG), in which it forms a plexus. The plexus gives rise to the five terminal branches of the facial nerve i.e. temporal, zygomatic, buccal, marginal mandibular and cervical. ${ }^{1}$

The temporal branch emerges from the superior border of the PG and crosses the zygomatic arch while the zygomatic branch passes via two or three branches superior and mainly inferior to the eye. ${ }^{1}$ The buccal branch passes superficial to the buccinator muscle to innervate this muscle and the

Dr. Norzana Abd Ghafar

Department of Anatomy

Faculty of Medicine,

Universiti Kebangsaan Malaysia Medical Centre,

Jalan Yaacob Latif, Bandar Tun Razak

56000 Cheras, Kuala Lumpur

Tel: +603-92898605

Fax: +60391458607

E-mail: norzana@ukm.edu.my marginal mandibular branch emerges from the inferior border of the PG and crosses the inferior border of the mandible. The cervical branch passes inferiorly to the inferior border of the PG and traverses a course posterior to the mandible to innervate the platysma muscle. ${ }^{1}$

The PG is the largest salivary gland and it is located anteriorly and inferiorly to the external acoustic meatus, partially covering the ramus of the mandible and the masseter muscle. ${ }^{2}$ The embryonic development of PG is well established. The PG is formed by the proliferating ectoderm of the primitive oral cavity, which invaginates into the adjacent mesenchyme during the sixth week of embryonic period. ${ }^{4}$ Following invagination, several epithelial sprouts proliferate and then migrate posteriorly in a chain of cells that bend over in order to form the ducts and this occurs by the tenth week of gestation. ${ }^{4}$

These ducts merge in increasing caliber until excretory ducts are formed, followed by larger ducts which finally merge to form a single main duct known as Stensen's duct. ${ }^{4}$ At the same time, the posterior ends of each epithelial structure differentiate to form acini, which begin to secrete by the eighteenth week of embryonic period. ${ }^{4}$ The Stensen's duct traverses a horizontal course to the anterior margin of the ramus of the mandible, passes laterally to the masseter, traverses around its anterior margin and pierces the buccinator muscle to open into the oral cavity. ${ }^{1}$

Proper knowledge of the normal topographic anatomy and variations of the Stensen's duct is highly relevant to clinical procedures including the analysis of radiographic images and computerized tomographic scans used in sialography, as well as for 
duct endoscopy, lithotripsy and trans-ductal facial nerve stimulation in the early stage of facial palsy.

In the present study, we highlight a rare case of anatomic variation of the parotid duct and anomalous branching of facial nerve which may be beneficial for diagnostic procedures and surgical interventions in day- to-day clinical practice.

\section{CASE REPORT}

During routine cadaveric dissection for anatomy postgraduates, a variation in the branching pattern of facial nerve was observed in a 50 -yearold male cadaver. The dissection was performed in layers, removing the skin, superficial fascia and subcutaneous fat. Then, the PG, duct and facial nerve branches were exposed. Facial nerve branching and numbers of rami of all the branches, anatomical relationship between its branches and adjacent structures in addition to a double parotid duct were documented and photographed.

On the right side, the facial nerve emerged from the anterior border of $P G$ branching in a unique pattern (Fig.1). From the most upper part of gland, temporal branch $(T)$ emerged running parallel to zygomatic arch. Zygomatic branch (Z1) emerged below the level of temporal branch to reach zygomaticus major muscle. Two roots emerged, one from upper part of the anterior border (R1) and the other (R2) from the middle part of the PG. Each root traversed a distance of $12 \mathrm{~mm}$ to unite $10 \mathrm{~mm}$ anterior to the PG and just below the parotid duct to form a semi-circle from which three branches emerged from above downwards. These were: accessory zygomatic branch (Z2), buccal branch (B) which in turn divided into upper and lower buccal divisions and marginal mandibular branch $(M)$. The last branch, cervical $(C)$, emerged from lower pole of $P G$ and then traversed towards the platysma muscle.

In the same cadaver, we also observed two parotid ducts which emerged from the anterior border of the PG. As shown in Fig 2, the first duct (D1) emerged from the upper one third of the anterior border and traversed horizontally for $9 \mathrm{~mm}$ to join the second duct. The second duct (D2) emerged from the middle one third of the anterior border of the gland and ran obliquely upwards and forwards for $19 \mathrm{~mm}$ to join D1 to form the main duct (D) at a point located $11 \mathrm{~mm}$ anterior to the PG. The main duct traversed a course over the masseter muscle for $4 \mathrm{~mm}$ then turned medially along the anterior border of the muscle to pierce the buccinator muscle. No abnormalities were observed on the left side.

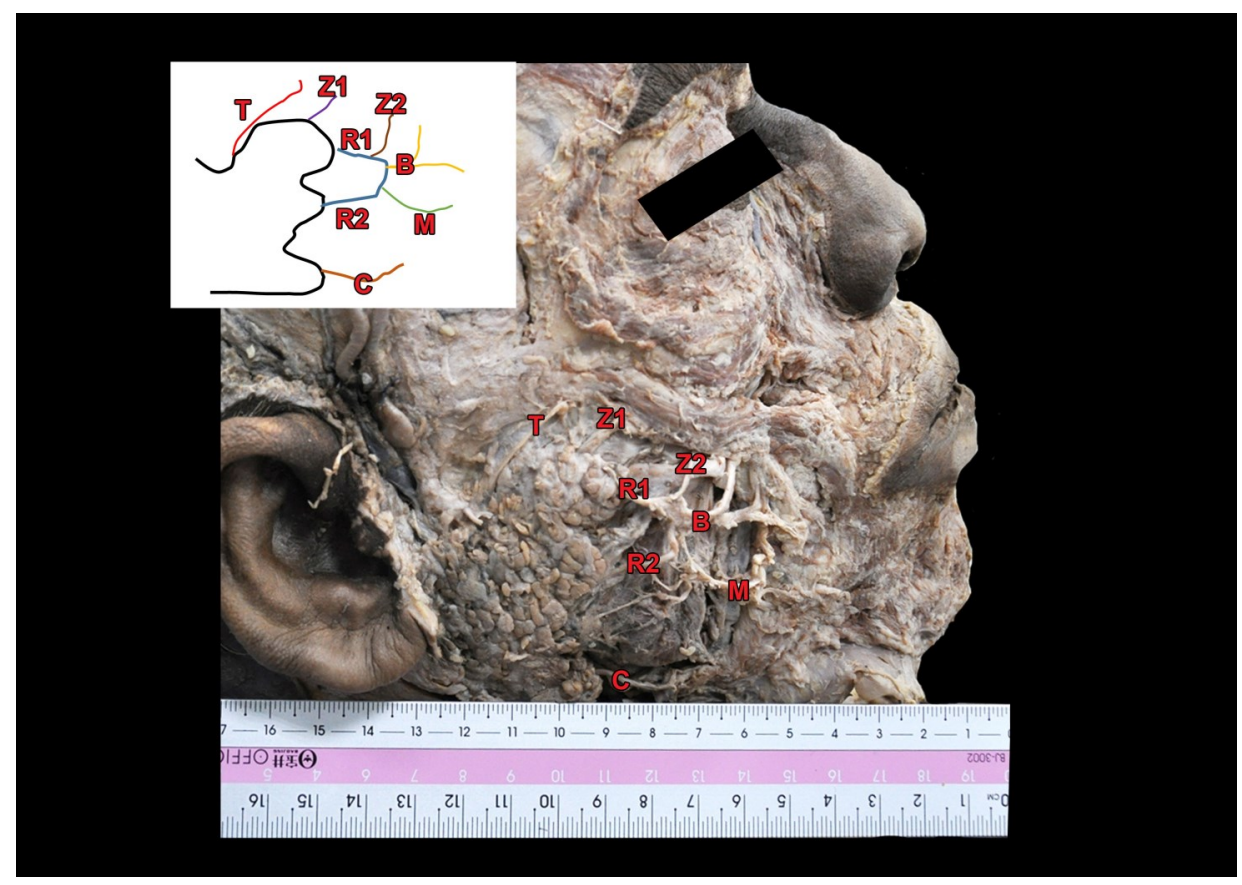

Fig.1. Photograph of dissected specimen and schematic diagram on the right side of the face showing the branching pattern of facial nerve
T: Temporal
Z1: Zygomatic
Z2: accessory zygomatic
B: Buccal
M: Mandibular
C: cervical
R1: $1^{\text {st }}$ root
R2: $2^{\text {nd }}$ root 


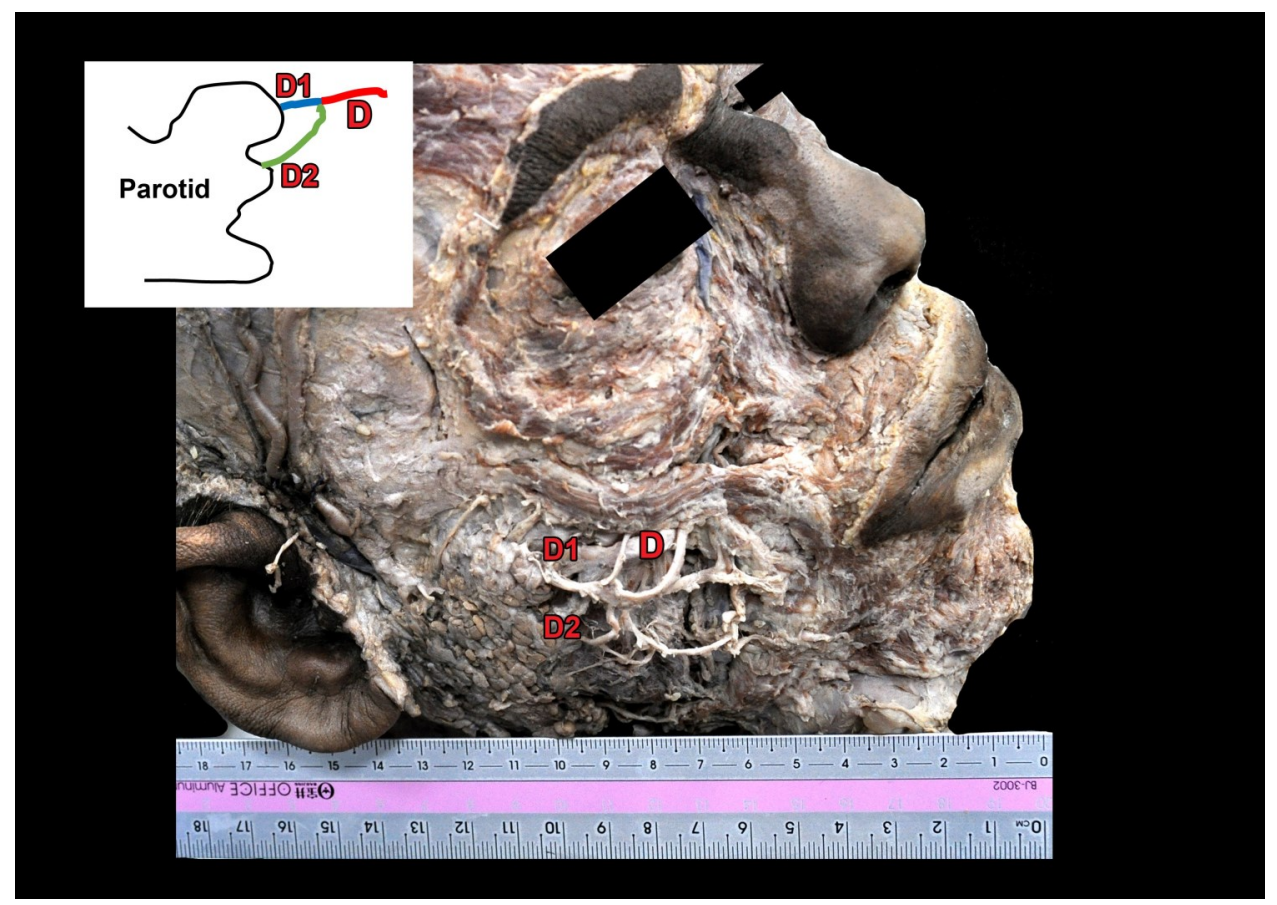

Fig.2. Photograph of dissected specimen and schematic diagram on the right side of the face showing the double parotid duct
D1: 1st duct
D2: 2nd duct
D: Main duct

\section{DISCUSSION}

The classification of the peripheral distribution of the facial nerve is based on the type and number of anastomoses between the peripheral branches. This was explained by Davis et al. who proposed that the pattern of branching of the facial nerve can be of 6 types, and found in their series that the types accounted for the following percentages: type I, 18\%; type II, 20\%; type III, 28\%; type IV, 24\%; type V, 9\%; and type VI, 6\%. Types III and IV were the most common and types II, III and IV made up $72 \%$ of all. ${ }^{3}$ The present case report showed the branching pattern of facial nerve which may not fit to any of the above types. Hence, the branching anomaly may be new of its kind.

Variations in branches of the facial nerve are best understood through its embryonic development. The main patterns of the nerve's complex course, branching pattern, and structural relationships are established during the first 3 months of prenatal life. ${ }^{5}$

When the crown-rump length (CRL) is about 21-22 $\mathrm{mm}$, the facial nerve reaches the future parotid space where it divides into the temporal and zygomatic branches. By the time the foetus has a CRL of $38-39 \mathrm{~mm}$; all peripheral branches of the facial nerve are identifiable. ${ }^{5}$

The anatomy of the parotid duct (Stensen's duct) is important for those professionals performing diagnostic and surgical procedures involving such anatomic structure. ${ }^{6}$ Aktan et al. (2001) and Fernandes et al. (2009) reported two cases of double parotid duct in the right aspect of the face in 63-year-old male human and 46-year-old male human subjects, respectively. ${ }^{7,8}$

We also observed double parotid duct in the right aspect of the face. However, we were not able to determine the features of each branch of this double duct (D1 and D2) inside the PG. Usually, the parotid duct traverses inferior to the zygomatic arch and then laterally to the masseter muscle at the anterior border of this muscle, it takes a medial turn to pierce the buccinator muscle to open into the crown of vestibule of the upper first or second molar crown. ${ }^{1,9}$

In the present case, the two parotid ducts united at a distance of $11 \mathrm{~mm}$ anterior to the PG to form a single duct and opened on the mucosa of the oral cavity. Aktan et al. (2001) also reported the merging of both branches before reaching the buccinator muscle but it was at a distance of $7 \mathrm{~mm}$ away from the muscle. ${ }^{7}$

The length of the ducts D1 and D2 in the present case was $9 \mathrm{~mm}$ and $19 \mathrm{~mm}$, respectively (Fig.2). Meanwhile, Aktan et al. (2001) reported a sole measurement - $55 \mathrm{~mm}$ while Fernandes et al. (2009) reported that the length of the ducts D1 and D2 were $26.49 \mathrm{~mm}$ and $37.25 \mathrm{~mm}$, respectively. ${ }^{7,8}$ Surgeons and radiologists performing any 
procedures in the region of the face should be aware of such anomalies pertaining to the PG and facial nerve.

\section{ACKNOWLEDGEMENT}

The authors wish to convey our sincere thanks to En. Mohd Rafizul Mohd Yusof, En. Fairuz Abd. Rashid, Pn. Siti Nor Ain Bakhtiar and Pn. Noor Azlina Zulkefli for their valuable help in dissection of the cadaver.

\section{REFERENCES}

1. Moore KL, Dalley AF. Clinically Oriented Anatomy. 5th edition. Toronto: Lippincott Williams \& Wilkins, 2014, 853 - 4 \& 914-6.

2. Standring S. Gray's Anatomy The anatomical basis of clinical practice. New York: Churchill Livingstone, 2008, 494-7.

3. Davis RA, Anson BJ, Budinger JM, Kurth LR. Surgical anatomy of the facial nerve and parotid gland based upon a study of 350 cervicofacial halves. Surg Gynecol Obstet 1956; 102:385-412.

4. Moore KL, Persaud TVN. The Developing Human: Clinically Oriented Embryology. Philadelphia: PA, 2013, 179.

5. Lee WT, Chung YY, Kim SW. A Morphological Analysis of the Facial Nerve in Korean Fetuses and Stillborn infants. J Korean Neurosurg Soc 2006; 40:445-9.

6. Samanta PP, Rana KK, Khan RQ, Das S. An unusually located human accessory parotid gland: A case report. Braz J Morphol Sci 2007; 24:53-4.

7. Aktan ZA, Bilge O, Pinar YA, Ikiz, AO. Duplication of the parotid duct: a previously unreported anomaly. Surg Radiol Anat 2001; 23:353-4.

8. Fernandes ACS, Lima GR, Rossi AM, Aguiar CM. Parotid gland with double duct: An anatomic variation description. Int. J. Morphol 2009; 27:129-132.

9. Gleeson M. Scott-Brown's Otolaryngology. 6th. London, Butterworth-Heinemann, Bath, 1997: V.1. p.3. 